03

\title{
Автомодельность в задаче о ламинарном пленочном кипении на вертикальной поверхности, погруженной в большой объем жидкости
}

\author{
() В.Б. Хабенский, А.А. Сулацкий
}

Научно-исследовательский технологический институт им. А.П. Александрова, 188540 Сосновый Бор, Ленинградская обл., Россия

e-mail: andrei314@mail.ru

Поступило в Редакцию 14 июля 2020 г.

В окончательной редакции 11 сентября 2020 г.

Принято к публикации 3 октября 2020 г.

С использованием методов автомодельности применительно к ламинарному свободноконвективному пленочному кипению на вертикальной поверхности конечной длины, погруженной в большой объем жидкости произвольного недогрева, получены выражения для критерия Нуссельта с непрерывным переходом от пленочного кипения в условиях насыщенной жидкости к условиям сильно недогретой жидкости как для теплообмена между поверхностью нагрева и межфазной границей, так и для теплоотдачи от этой границы в объем жидкости, причем с одновременным учетом теплообмена излучением. Показано наличие в данной задаче иерархии автомодельностей различного рода.

Ключевые слова: автомодельность, пленочное кипение, вертикальная поверхность, недогрев.

DOI: 10.21883/JTF.2021.03.50516.226-20

\section{Введение}

Пленочное кипение является одной из наиболее детально исследованных задач термогидродинамики двухфазных сред. Перечислить все или даже только основные работы, посвященные данной проблеме, не представляется возможным. Актуальное состояние проблемы достаточно подробно освещается в обзорах [1-5]. Однако в силу именно этой „исчерпывающей изученности“, указанная задача является хорошим объектом для демонстрации применения перспективных математических методов исследования.

В последние несколько десятилетий расчетные, компьютерные методы существенно потеснили теоретикоаналитические (как, впрочем, и экспериментальные) методы исследований, причем часто это происходит в ущерб пониманию процессов, в то время как именно теоретико-аналитические методы позволяют выявить физику процесса, определить зависимость его от тех или иных параметров задачи, получить важные следствия и помочь в интерпретации численных результатов, которые при одном лишь расчетном исследовании могут быть не вполне очевидными.

Одними из эффективных методов теоретического исследования разнообразных проблем физики, но преимущественно считающихся второстепенными, вспомогательными, являются методы, основанные на теории размерности, подобия и автомодельности. Как известно, такие методы ранее применялись достаточно широко: от задач гидрогазодинамики, теории горения и астрофизики до разнообразных областей биологии. Впервые понятие автомодельности сформулировал А.А. Гухман в
1928 г. [6,7]. Дальнейшее развитие идей автомодельности было осуществлено многими исследователями, такими как Я.Б. Зельдович, Ю.П. Райзер, К.В. Брушлинский, Я.М. Каждан, Г.И. Баренблатт [8-11] и др. В монографии Баренблатта [11] предложена простая и ясная классификация видов автомодельности, и приведены многочисленные примеры. В частности, в [11] были даны точные определения полной автомодельности по параметру(ам) (автомодельность 1-го рода) и неполной автомодельности по параметру(ам) (автомодельность 2-го рода). Согласно [11], полной автомодельностью (автомодельностью 1-го рода) называется ситуация, когда при стремлении некоторого параметра задачи к нулю или бесконечности решение задачи стремится к конечному, отличному от нуля, или бесконечности решению. Первоначально введенное в [6] понятие автомодельности в терминах [11] соответствует именно полной автомодельности. В случае неполной автомодельности (автомодельности 2-го рода) по параметру (для определенности по параметру $\left.\Pi_{1}\right)$ искомая функция решения задачи (П) может стремиться к нулю, к бесконечности или вообще не стремиться ни к какому пределу, но имеет при малом или, напротив, большом $\Pi_{1}$ степенную асимптотику:

$$
\Pi=\Phi\left(\Pi_{1}, \Pi_{2}, \ldots\right)=\Pi_{1}^{\alpha} \Phi_{1}\left(\Pi_{2} / \Pi_{1}^{\beta}, \ldots\right),
$$

где степени $\alpha$ и $\beta$ определяются из дополнительных соображений. Если в случае автомодельности 1-го рода для получения полезных следствий и результатов часто (но далеко не всегда) достаточно простого анализа размерности, то в случае автомодельности 2-го рода такой минималистский подход недостаточен - требуется анализ описывающих конкретный физический процесс 
системы уравнений, их начальных и/или граничных условий, причем сам способ получения подобных степенных асимптотик следует непосредственно из определения (1). Здесь и далее используется терминология, принятая в [11].

В настоящей работе, отнюдь не игнорируя ранее проведенные исследования пленочного кипения, демонстрируются возможности методов автомодельности (1-го и 2-го рода) на примере ламинарного свободноконвективного пленочного кипения на вертикальной поверхности конечной длины, погруженной в большой объем жидкости произвольного недогрева. При этом впервые оказалось возможным получить выражения для критерия Нуссельта с непрерывным переходом от пленочного кипения в условиях насыщенной жидкости к условиям сильно недогретой жидкости как для теплообмена между поверхностью нагрева и межфазной границей, так и для теплоотдачи от этой границы в объем жидкости, причем с одновременным учетом теплообмена излучением.

\section{1. Приближения математической модели, система уравнений и граничные условия}

Схема процесса показана на рисунке.

Приближения математической модели процесса следующие:

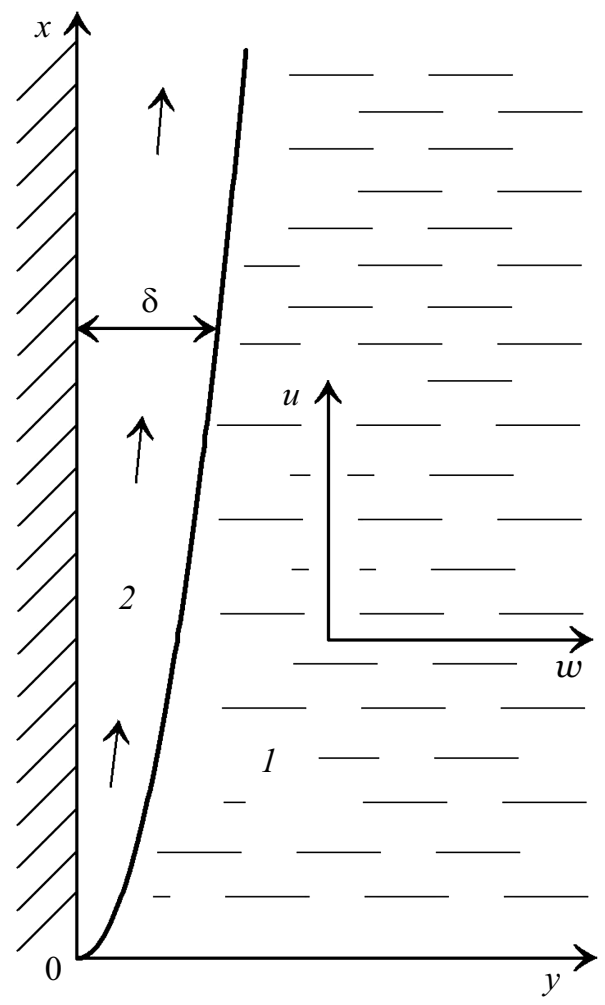

Схема свободноконвективного пленочного кипения на вертикальной поверхности, погруженной в большой объем жидкости: 1 - жидкость, 2 - пар.
1. Как для жидкости, так и для пара применимо приближение ламинарного пограничного слоя.

2. Движение пара в пленке вызывается архимедовой силой, обусловленной разницей плотностей жидкости и пара.

3. Как жидкость, так и пар несжимаемы, но имеет место термическое расширение жидкости. Влиянием термического расширения пара пренебрегается.

4. Рассматривается стационарный случай.

Система уравнений имеет вид:

$$
\begin{gathered}
\frac{\partial u_{1}}{\partial x}+\frac{\partial w_{1}}{\partial y}=0 \\
u_{1} \frac{\partial u_{1}}{\partial x}+w_{1} \frac{\partial u_{1}}{\partial y}=\beta_{1} \cdot\left(T_{1}-T_{1, \infty}\right) g+v_{1} \frac{\partial^{2} u_{1}}{\partial y^{2}}, \\
u_{1} \frac{\partial T_{1}}{\partial x}+w_{1} \frac{\partial T_{1}}{\partial y}=\frac{\lambda_{1}}{\rho_{1} c_{p, 1}} \cdot \frac{\partial^{2} T_{1}}{\partial y^{2}} \\
\frac{\partial u_{2}}{\partial x}+\frac{\partial w_{2}}{\partial y}=0 \\
u_{2} \frac{\partial u_{2}}{\partial x}+w_{2} \frac{\partial u_{2}}{\partial y}=\frac{\Delta \rho g}{\rho_{2}}+v_{2} \frac{\partial^{2} u_{2}}{\partial y^{2}} \\
u_{2} \frac{\partial T_{2}}{\partial x}+w_{2} \frac{\partial T_{2}}{\partial y}=\frac{\lambda_{2}}{\rho_{2} c_{p, 2}} \cdot \frac{\partial^{2} T_{2}}{\partial y^{2}}
\end{gathered}
$$

где $x, y$ - декартовы координаты (см. рисунок), [m]; $u_{j}, w_{j}$ - компоненты скорости $j$-й фазы вдоль декартовых осей и соответственно (см. рисунок), $[\mathrm{m} / \mathrm{s}]$; $T_{j}$ - температура $j$-й фазы, $[\mathrm{K}] ; \beta_{1}-$ коэффициент термического расширения жидкости, $[1 / \mathrm{K}], g$ - ускорение свободного падения, $[\mathrm{m} / \mathrm{s}]^{2} ; v_{j}-$ кинематическая вязкость $j$-й фазы, $\left[\mathrm{m}^{2} / \mathrm{s}\right] ; \lambda_{j}-$ теплопроводность $j$-й фазы, $[\mathrm{W} /(\mathrm{m} \cdot \mathrm{K})] ; c_{p, j}-$ удельная теплоемкость $j$-й фазы, $[\mathrm{J} /(\mathrm{kg} \cdot \mathrm{K})] ; \rho_{j}$ - плотность $j$-й фазы, $\left[\mathrm{kg} / \mathrm{m}^{3}\right]$; $\Delta \rho=\rho_{1}-\rho_{2}$; индекс $j-, 1$, и „2“, жидкость и пар соответственно.

Граничные условия:

1) поверхность нагрева $\left(y=0\right.$, индекс „, $\left.w^{6 \varsigma}\right)$ :

- условие прилипания : $u_{2, w}=0$,

- условие непроницаемости : $w_{2, w}=0$,

- условие изотермичности : $\quad T_{2, w}=T_{w}=\mathrm{const} ;(10)$

2) межфазная граница $(y=\delta(x)$, индекс ,,i“):

- равенство массовых потоков через границу фаз:

$\rho_{2}\left[w_{2, i}-u_{2, i} \cdot(d \delta / d x)\right]=\rho_{1}\left[w_{1, i}-u_{1, i} \cdot(d \delta / d x)\right]$

- равенство касательных границе раздела фаз состав-

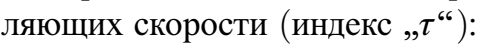

$w_{2, \tau}=w_{1, \tau} \Leftrightarrow u_{2, i}+w_{2, i} \cdot(d \delta / d x)=u_{1, i}+w_{1, i} \cdot(d \delta / d x)$, 
— равенство касательных границе раздела фаз напряжений:

$$
\begin{aligned}
\mu_{2} \frac{\partial w_{2, \tau}}{\partial n} & =\mu_{1} \frac{\partial w_{1, \tau}}{\partial n} \Leftrightarrow \mu_{2}\left[\frac{\partial u_{2}}{\partial y}+\left(\frac{\partial w_{2}}{\partial y}-\frac{\partial u_{2}}{\partial x}\right) \cdot \frac{d \delta}{d x}\right] \\
& =\mu_{1}\left[\frac{\partial u_{1}}{\partial y}+\left(\frac{\partial w_{1}}{\partial y}-\frac{\partial u_{1}}{\partial x}\right) \cdot \frac{d \delta}{d x}\right]
\end{aligned}
$$

- связь массового потока через межфазную границу с подводимым к ней со стороны пара и отводимым от нее в жидкость тепловым потоком:

$$
\begin{aligned}
& \left(-\lambda_{2} \frac{\partial T_{2}}{\partial n}+q_{r}\right)_{i}-\left(-\lambda_{1} \frac{\partial T_{1}}{\partial n}\right)_{i}=-\rho_{2} \Delta h \\
& \times\left(w_{2, i}-u_{2, i} \frac{d \delta}{d x}\right) \Leftrightarrow-\lambda_{2}\left(\frac{\partial T_{2}}{\partial y}-\frac{\partial T_{2}}{\partial x} \cdot \frac{d \delta}{d x}\right)_{i} \\
& +\varepsilon \sigma_{S B} \cdot\left(T_{w}^{4}-T_{s}^{4}\right)+\lambda_{1}\left(\frac{\partial T_{1}}{\partial y}-\frac{\partial T_{1}}{\partial x} \cdot \frac{d \delta}{d x}\right)_{i} \\
& =-\rho_{2} \Delta h \cdot\left(w_{2, i}-u_{2, i} \frac{d \delta}{d x}\right),
\end{aligned}
$$

где $\Delta h-$ скрытое тепло парообразования,

- равенство температуры межфазной границы температуре насыщения жидкости:

$$
T_{2, i}=T_{1, i}=T_{s}
$$

3) объем жидкости $\left(y \rightarrow \infty\right.$, индекс „, $\left.\infty^{6}\right)$ :

$$
\begin{array}{ll}
\text { - неподвижность жидкости: } & u_{1, \infty}=0, \\
\text { — изотермичность жидкости: } & T_{1, \infty}=T_{\infty} .
\end{array}
$$

От скоростей жидкости и газа перейдем к соответствующим функциям тока:

$$
u_{j}=\partial \Phi_{j} / \partial y, \quad w_{j}=-\partial \Phi_{j} / \partial x, \quad j=1,2,
$$

после чего система уравнений и граничных условий примет вид:

$$
\begin{gathered}
\frac{\partial \Phi_{1}}{\partial y} \cdot \frac{\partial^{2} \Phi_{1}}{\partial x \partial y}-\frac{\partial \Phi_{1}}{\partial x} \cdot \frac{\partial^{2} \Phi_{1}}{\partial y^{2}}=\beta_{1} \cdot\left(T_{1}-T_{1, \infty}\right) g+v_{1} \frac{\partial^{3} \Phi_{1}}{\partial y^{3}} \\
\frac{\partial \Phi_{1}}{\partial y} \cdot \frac{\partial T_{1}}{\partial x}-\frac{\partial \Phi_{1}}{\partial x} \cdot \frac{\partial T_{1}}{\partial y}=\frac{\lambda_{1}}{\rho_{1} c_{p, 1}} \cdot \frac{\partial^{2} T_{1}}{\partial y^{2}} \\
\frac{\partial \Phi_{2}}{\partial y} \cdot \frac{\partial^{2} \Phi_{2}}{\partial x \partial y}-\frac{\partial \Phi_{2}}{\partial x} \cdot \frac{\partial^{2} \Phi_{2}}{\partial y^{2}}=\frac{\Delta \rho g}{\rho_{2}}+v_{2} \frac{\partial^{3} \Phi_{2}}{\partial y^{3}} \\
\frac{\partial \Phi_{2}}{\partial y} \cdot \frac{\partial T_{2}}{\partial x}-\frac{\partial \Phi_{2}}{\partial x} \cdot \frac{\partial T_{2}}{\partial y}=\frac{\lambda_{2}}{\rho_{2} c_{p, 2}} \cdot \frac{\partial^{2} T_{2}}{\partial y^{2}}
\end{gathered}
$$

Уравнения неразрывности (2) и (5) при этом удовлетворяются тождественно.

Ввиду того, что с вводом функций тока был осуществлен переход к системе дифференциальных уравнений на порядок большей, чем исходная система (2)-(7), требуются 2 дополнительных граничных условия для $\Phi_{1}$ и $\Phi_{2}$. Отметим, что, поскольку функции тока являются неизмеримыми параметрами процесса, данные дополнительные граничные условия можно вводить достаточно произвольно, например, из соображений упрощения решения.

Тогда граничные условия примут вид:

1) поверхность нагрева:

- дополнительное условие для пара: $\Phi_{2, w}=0$,

- условие прилипания: $\left(\partial \Phi_{2} / \partial y\right)_{w}=0$,

- условие непроницаемости: $\left(\partial \Phi_{2} / \partial x\right)_{w}=0$,

- условие изотермичности: $T_{2, w}=T_{w}=\mathrm{const}$;

2) межфазная граница:

-дополнительное условие для жидкости будет определено позже;

- равенство массовых потоков через границу пара и жидкости:

$$
\rho_{1} \cdot\left(\frac{\partial \Phi_{1}}{\partial x}+\frac{\partial \Phi_{1}}{\partial y} \cdot \frac{d \delta}{d x}\right)_{i}=\rho_{2} \cdot\left(\frac{\partial \Phi_{2}}{\partial x}+\frac{\partial \Phi_{2}}{\partial y} \cdot \frac{d \delta}{d x}\right)_{i},
$$

- равенство касательных составляющих скорости:

$$
\left(\frac{\partial \Phi_{1}}{\partial y}-\frac{\partial \Phi_{1}}{\partial x} \cdot \frac{d \delta}{d x}\right)_{i}=\left(\frac{\partial \Phi_{2}}{\partial y}-\frac{\partial \Phi_{2}}{\partial x} \cdot \frac{d \delta}{d x}\right)_{i}
$$

- равенство касательных напряжений:

$\mu_{1}\left(\frac{\partial^{2} \Phi_{1}}{\partial y^{2}}-2 \frac{\partial^{2} \Phi_{1}}{\partial x \partial y} \cdot \frac{d \delta}{d x}\right)_{i}=\mu_{2}\left(\frac{\partial^{2} \Phi_{2}}{\partial y^{2}}-2 \frac{\partial^{2} \Phi_{2}}{\partial x \partial y} \cdot \frac{d \delta}{d x}\right)_{i}$,

- связь массового потока через межфазную границу с подводимым к ней со стороны пара и отводимым от нее в жидкость тепловым потоком:

$$
\begin{aligned}
& -\lambda_{2} \cdot\left(\frac{\partial T_{2}}{\partial y}-\frac{\partial T_{2}}{\partial x} \cdot \frac{d \delta}{d x}\right)_{i}+\varepsilon \sigma_{S B} \cdot\left(T_{w}^{4}-T_{s}^{4}\right)+\lambda_{1} \\
& \times\left(\frac{\partial T_{1}}{\partial y}-\frac{\partial T_{1}}{\partial x} \cdot \frac{d \delta}{d x}\right)_{i}=\rho_{2} \Delta h \cdot\left(\frac{\partial \Phi_{2}}{\partial x}+\frac{\partial \Phi_{2}}{\partial y} \cdot \frac{d \delta}{d x}\right)_{i},
\end{aligned}
$$

- равенство температуры межфазной границы температуре насыщения жидкости:

$$
T_{1, i}=T_{2, i}=T_{s}
$$

3) объем жидкости:

$$
\begin{aligned}
& \text { - неподвижность жидкости: }\left(\partial \Phi_{1} / \partial y\right)_{\infty}=0, \\
& \text { — изотермичность жидкости: } T_{1, \infty}=T_{\infty} .
\end{aligned}
$$




\section{2. Полная автомодельность (автомодельность 1-го рода) задачи по параметру соотношения подъемных сил в жидкости и в паре}

Введем безразмерные обозначения:

- безразмерные координаты:

$$
\eta_{1}=\sqrt{v_{2} / v_{1}} \cdot y /\left(\Lambda^{3 / 2} x^{1 / 4}\right), \quad \eta_{2}=y /\left(\Lambda^{3 / 2} x^{1 / 4}\right)
$$

— безразмерные функции тока:

$$
\begin{gathered}
\left.f_{1}\left(\eta_{1}\right)=\sqrt{v_{2} / v_{1}}\right) \cdot(\Lambda / x)^{3 / 4} \cdot\left[\left(3 \Phi_{1}\right) /\left(4 v_{2}\right)\right] \\
f_{2}\left(\eta_{2}\right)=(\Lambda / x)^{3 / 4} \cdot\left[\left(3 \Phi_{2}\right) /\left(4 v_{2}\right)\right]
\end{gathered}
$$

- безразмерные температуры:

$$
\begin{gathered}
\Theta_{1}\left(\eta_{1}\right)=\left(T_{1}-T_{\infty}\right) /\left(T_{s}-T_{\infty}\right), \\
\Theta_{2}\left(\eta_{2}\right)=\left(T_{2}-T_{s}\right) /\left(T_{w}-T_{s}\right), \\
\text { где } \Lambda=\left[\left(4 \rho_{2} v_{2}^{2}\right) /(3 \Delta \rho g)\right]^{1 / 3}
\end{gathered}
$$

- характерный линейный (пространственный), т. е. имеющий размерность длины, масштаб взаимодействия вязкости пара и архимедовой силы. Подобные переменные, отличающиеся от введенных здесь только безразмерными множителями, вводились ранее в работах $[12,13]$. Связь введенных в [12,13] переменных (обозначены

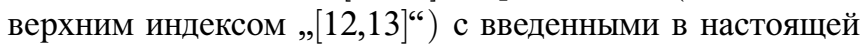
работе (обозначения без верхних индексов) следующая:

$$
\begin{gathered}
\eta_{1}^{[12,13]}=3^{-1 / 4}\left[\rho_{2} \beta_{1} \cdot\left(T_{s}-T_{\infty}\right) / \Delta \rho\right]^{1 / 4} \eta_{1}, \\
\eta_{2}^{[12,13]}=3^{-1 / 4} \eta_{2}, \\
f_{1}^{[12,13]}=3^{-3 / 4}\left\{\Delta \rho /\left[\rho_{2} \beta_{1} \cdot\left(T_{s}-T_{\infty}\right)\right]\right\}^{1 / 4} f_{1}, \\
f_{2}^{[12,13]}=3^{-3 / 4} f_{2} .
\end{gathered}
$$

Отметим, что для сокращения, упрощения выражений знак производной любого порядка (верхний штрих) любой $j$-й функции будет означать производную соответствующего порядка по $j$-й же координате.

Уравнение движения жидкости (19) в новых переменных примет вид

$$
\begin{aligned}
& f_{1}^{\prime \prime \prime}+f_{1} f_{1}^{\prime \prime}-(2 / 3) \cdot\left(f_{1}^{\prime}\right)^{2} \\
& +\left[\rho_{2} \beta_{1} \cdot\left(T_{s}-T_{1, \infty}\right) / \Delta \rho\right] \cdot \Theta_{1}=0 .
\end{aligned}
$$

Стоящий в 4-м слагаемом (38) перед $\Theta_{1}$ безразмерный множитель $\rho_{2} \beta_{1} \cdot\left(T_{s}-T_{1, \infty}\right) / \Delta \rho$ представляет собой соотношение подъемной силы в жидкости, вызванной ее термическим расширением $\left(\rho_{1} \beta_{1} \cdot\left(T_{s}-T_{1, \infty}\right) g\right)$, и действующей на пар архимедовой силы $(\Delta \rho g)$, умноженное, к тому же, на малый симплекс плотностей пара и жидкости $\left(\rho_{2} / \rho_{1}\right)$. Легко оценить, что весь этот множитель в целом - весьма мал $(\ll 1)$. При этом все остальные 3 слагаемых уравнения (38) - порядка единицы. Поэтому 4-м слагаемым в (38) можно пренебречь без вырождения этого уравнения в бессодержательное математическое выражение. С физической точки зрения это означает, что поле скоростей в жидкости определяется условиями динамического взаимодействия пара и жидкости на границе раздела фаз и соответственно опосредованно скоростью пара в паровой пленке, но не силами плавучести в жидкости. Таким образом, уравнение движения жидкости (38) принимает вид

$$
f_{1}^{\prime \prime \prime}+f_{1} f_{1}^{\prime \prime}-(2 / 3) \cdot\left(f_{1}^{\prime}\right)^{2}=0 .
$$

В новых обозначениях уравнения (20)-(22) примут вид соответственно

$$
\begin{gathered}
\Theta_{1}^{\prime \prime}+\operatorname{Pr}_{1} f_{1} \Theta_{1}^{\prime}=0, \\
f_{2}^{\prime \prime \prime}+f_{2} f_{2}^{\prime \prime}-(2 / 3) \cdot\left(f_{2}^{\prime}\right)^{2}+1=0 . \\
\Theta_{2}^{\prime \prime}+P r_{2} f_{2} \Theta_{2}^{\prime}=0 .
\end{gathered}
$$

Перед тем как записать граничные условия в новых переменных, запишем выражения для координат $\eta_{1}$ и $\eta_{2}$ на межфазной границе

$$
\begin{gathered}
\eta_{1}=\eta_{1, i}=\sqrt{v_{2} / v_{1}} \cdot \delta(x) /\left(\Lambda^{3 / 4} x^{1 / 4}\right) \\
\eta_{2}=\eta_{2, i}=\delta(x) /\left(\Lambda^{3 / 4} x^{1 / 4}\right)
\end{gathered}
$$

Граничные условия:

1) поверхность нагрева $\left(\eta_{2}=0\right)$ :

$$
f_{2, w}=0, \quad f_{2, w}^{\prime}=0, \quad \Theta_{2, w}=1
$$

Отметим, что условие непроницаемости $\left(f_{2, w}-\eta_{2, w} f_{2, w}^{\prime} / 3=0\right)$ выполняется тождественно, так как все три переменные в выражении этого условия равны нулю, т.е. из числа граничных условий оно исключается;

2) межфазная граница $\left(\eta_{1}=\eta_{1, i}\right.$ и $\left.\eta_{2}=\eta_{2, i}\right)$ :

- равенство массовых потоков через границу фаз

$$
\begin{aligned}
& f_{1, i}-\left(\eta_{1, i} f_{1, i}^{\prime} / 3\right) \cdot[1-(4 x / \delta) \cdot(d \delta / d x)] \\
& =R \cdot\left\{f_{2, i}-\left(\eta_{2, i} f_{2, i}^{\prime} / 3\right) \cdot[1-(4 x / \delta) \cdot(d \delta / d x)]\right\}, \\
& \text { где введен параметр } \quad R=\left(\rho_{2} / \rho_{1}\right) \cdot \sqrt{v_{2} / v_{1}} ;
\end{aligned}
$$

- равенство касательных составляющих скорости

$$
\begin{aligned}
& 4 f_{1, i}^{\prime}-\left(3 f_{1, i} / \eta_{1, i}-f_{1, i}^{\prime}\right) \cdot(\delta / x) \cdot(d \delta / d x) \\
& =4 f_{2, i}^{\prime}-\left(3 f_{2, i} / \eta_{2, i}-f_{2, i}^{\prime}\right) \cdot(\delta / x) \cdot(d \delta / d x) .
\end{aligned}
$$

Учитывая, что неравенство $\delta \ll x$ начинает выполняться уже на очень малых длинах обогреваемой поверхности $x$, предполагая, что $|d \delta / d x| \ll 1$ и тем более $(\delta / x) \cdot|d \delta / d x| \ll 1$, можно упростить (47)

$$
f_{1, i}^{\prime}=f_{2, i}^{\prime}
$$


- условия (29), (30) с учетом малости $(\delta / x) \cdot(d \delta / d x)$ преобразуются к виду

$$
\begin{gathered}
f_{1, i}^{\prime \prime}=R f_{2, i}^{\prime \prime}, \\
\frac{K_{1}}{P r_{1}} \Theta_{1, i}^{\prime}-R \cdot \frac{K_{2}}{P r_{2}} \Theta_{2, i}^{\prime}+R B \cdot\left(\frac{x}{L}\right)^{1 / 4} \\
=R \cdot\left[f_{2, i}-\frac{\eta_{2, i} f_{2, i}^{\prime}}{3} \cdot\left(1-\frac{4 x}{\delta} \cdot \frac{d \delta}{d x}\right)\right],
\end{gathered}
$$

где введены числа Прандтля $\left(P r_{1}, P r_{2}\right)$ и числа Кирпичева-Кутателадзе для жидкости и пара:

$$
K_{1}=c_{p, 1}\left(T_{s}-T_{1, \infty} / \Delta h\right), \quad K_{2}=c_{p, 2}\left(T_{w}-T_{s} / \Delta h\right),
$$

а также введен

$$
B=\left[\varepsilon \sigma_{S B} \cdot\left(T_{w}^{4}-T_{s}^{4}\right) /\left(\rho_{2} \Delta h\right)\right] \cdot\left[4 \rho_{2} L /\left(3 \Delta \rho g v_{2}^{2}\right)\right]^{1 / 4}
$$

- безразмерный комплекс, с точностью до постоянного множителя представляющий собой величину, обратную числу Больцмана (критерию конвективно-радиационного теплообмена), вычисленного по характерной для данной задачи величине скорости свободной конвекции: $\left(g \Delta \rho v_{2}^{2} / \rho_{2} L\right)^{1 / 4}$, где $L-$ высота поверхности нагрева.

$$
\text { — температурные условия: } \Theta_{2, i}=0, \quad \Theta_{1, i}=1 \text {; }
$$

3) объем жидкости $\left(\eta_{1} \rightarrow \infty\right)$ :

$$
f_{1, \infty}^{\prime}=0, \quad \Theta_{1, \infty}=0
$$

Видно, что ни в одном из граничных условий, ни в одном из дифференциальных уравнений системы, кроме уравнения движения жидкости, комплекс, характеризующий соотношение подъемных сил в жидкости и в паре $\left(\rho_{2} \beta_{1} \cdot\left(T_{s}-T_{1, \infty}\right) / \Delta \rho\right)$, не появляется, и соответственно после удаления из уравнения движения жидкости слагаемого с этим комплексом (что соответствует математической процедуре $\left.\rho_{2} \beta_{1} \cdot\left(T_{s}-T_{1, \infty}\right) / \Delta \rho \rightarrow 0\right)$ этот параметр уже никак не влияет на решение. А поскольку это решение получается не нулевым (и не бесконечным), что подтверждается результатами проведенного авторами численного решения задачи по методике [12] (результаты которого здесь не приводятся), мы имеем полную автомодельность (автомодельность 1-го рода) по данному параметру. Отметим, что точный вид этого параметра из простого анализа размерности определить было бы невозможно.

Также отметим, что полученная система уравнений и граничных условий остается не автомодельной по физической координате х, так как в тепловом условии на межфазной границе (50) присутствуют в явном виде и сама координата, и ее функции: $\delta(x)$ и $d \delta / d x$.

\section{3. Неполная автомодельность (автомодельность 2-го рода) задачи по параметру $R$}

Из определения параметра $R(46)$ легко понять, что его характерная величина много меньше единицы: $R \ll 1$. Однако поступить так же, как это было сделано с комплексом соотношения в фазах сил плавучести, т. е. исключив из граничных условий (в дифференциальных уравнениях параметр $R$ отсутствует) все слагаемые с $R$, нельзя. Во-первых, при этом исчезнет единственное слагаемое, ответственное за радиационный теплообмен 3-е слагаемое в условии (50). Во-вторых, и это главное, при прямом предельном переходе $R \rightarrow 0$ решение исходной системы не стремится к конечному пределу. Условия на границе раздела фаз (49) и (50) при $R \rightarrow 0$ приводят к бесконечно большим значениям касательного напряжения и теплового потока у этой границы со стороны пара. Так как в физических задачах бесконечности невозможны и являются математическими артефактами, адекватное математическое решение исключает допущение о равенстве $R$ нулю. Таким образом, полной независимости решения задачи от параметра $R$ нет, и его влияние остается существенным, как бы ни мала была его величина. Согласно [11], в подобных случаях возможно искать решение в виде степенных асимптотик (1).

Введем новые безразмерные координаты и функции тока $\left(\xi_{1}, \xi_{2}, \Psi_{1}, \Psi_{2}\right)$ следующим образом:

$$
\begin{gathered}
\eta_{1}=R^{\alpha_{1 T}} \xi_{1}, \quad \eta_{2}=R^{\alpha_{2 T}} \xi_{2}, \\
f_{1}=R^{\alpha_{1 \Psi}} \Psi_{1}, \quad f_{2}=R^{\alpha_{2 \Psi}} \Psi_{2} .
\end{gathered}
$$

Подставим соотношения (55) сначала в уравнения системы (39)-(42). Штрихи в производных теперь будут обозначать производные по $\xi_{1}$ и $\xi_{2}$ в уравнениях для жидкости и пара соответственно. Получим

$$
\begin{gathered}
R^{\alpha_{1 \Psi}-3 \alpha_{1 T}} \Psi_{1}^{\prime \prime \prime}+R^{2 \alpha_{1 \Psi}-2 \alpha_{1 T}} \Psi_{1} \Psi_{1}^{\prime \prime} \\
-R^{2 \alpha_{1 \Psi}-2 \alpha_{1 T}} \cdot(2 / 3) \cdot\left(\Psi_{1}^{\prime}\right)^{2}=0, \\
R^{-2 \alpha_{1 T}} \Theta_{1}^{\prime \prime}+R^{\alpha_{1 \Psi}-\alpha_{1 T}} \operatorname{Pr}_{1} \Psi_{1} \Theta_{1}^{\prime}=0, \\
R^{\alpha_{2 \Psi}-3 \alpha_{2 T}} \Psi_{2}^{\prime \prime \prime}+R^{2 \alpha_{2 \Psi}-2 \alpha_{2 T}} \Psi_{2} \Psi_{2}^{\prime \prime} \\
-R^{2 \alpha_{2 \Psi}-2 \alpha_{2 T}} \cdot(2 / 3) \cdot\left(\Psi_{2}^{\prime}\right)^{2}+R^{0}=0, \\
R^{-2 \alpha_{2 T}} \Theta_{2}^{\prime \prime}+R^{\alpha_{2 \Psi}-\alpha_{2 T}} \operatorname{Pr}_{2} \Psi_{2} \Theta_{2}^{\prime}=0 .
\end{gathered}
$$

Для того чтобы уравнение движения жидкости (56) не стало физически бессодержательным, вводится очевидное предположение о том, что силы вязкости (1-е слагаемое) уравновешены силами инерции (2-е и 3-е слагаемые), т. е. указанные силы имеют величину одного порядка. То есть степени при $R$ в 1-м слагаемом, с одной стороны, и во 2-м и 3-м, с другой, должны быть равны

$$
\alpha_{1 \Psi}-3 \alpha_{1 T}=2 \alpha_{1 \Psi}-2 \alpha_{1 T} \Rightarrow \alpha_{1 \Psi}=-\alpha_{1 T} .
$$


Точно такое же соотношение между $\alpha_{1 \Psi}$ и $\alpha_{1 T}$ следует из (57) из соображений, что в жидкости теплопроводность должна быть одного порядка величины с конвективным переносом тепла.

В уравнении движения жидкости (58) помимо слагаемых, ответственных за вязкие силы (1-е слагаемое) и силы инерции (2-е и 3-е слагаемые), присутствует слагаемое, ответственное за архимедову силу (4-е слагаемое). Архимедова сила - единственный источник движения при свободноконвективном пленочном кипении. Поэтому это слагаемое, безусловно, должно остаться в уравнении (58). С другой стороны, из обширной экспериментальной и теоретической практики известно, что основные силы, препятствующие движению в паровой пленке, - это силы вязкости (1-е слагаемое). Поэтому в (58) должны быть равны степени при $R$ в 1-м слагаемом (вязкие силы) и в последнем, 4-м, слагаемом (сила Архимеда):

$$
\alpha_{2 \Psi}-3 \alpha_{2 T}=0 \Rightarrow \alpha_{2 \Psi}=3 \alpha_{2 T} .
$$

Зная (61) и учитывая сделанное выше предположение, что 2-е и 3-е слагаемые (силы инерции) в уравнении (58) должны быть много меньше, чем 1-е и 4-е слагаемые, получим дополнительное условие для $\alpha_{2 \Psi}$ и $\alpha_{2 T}$ :

$$
2 \alpha_{2 \Psi}-2 \alpha_{2 T}>0 \Rightarrow 2 \cdot\left(3 \alpha_{2 T}\right)-2 \alpha_{2 T}>0 \Rightarrow \alpha_{2 T}>0 .
$$

Подстановка (61) в (59) и учет неравенства (62) приводят к тому, что в тепловом уравнении для пара теплопроводность (1-е слагаемое) оказывается много больше переноса тепла за счет конвекции (2-е слагаемое), что вполне ожидаемо для тонких слоев среды.

Подставим (55), (60) и (61) в массовое, кинематическое и динамическое граничные условия на границе раздела жидкости и пара, т.е. в равенства (45), (48) и (49) соответственно

$$
\begin{gathered}
\Psi_{1, i}-\left(\xi_{1, i} \Psi_{1, i}^{\prime} / 3\right) \cdot[1-(4 x / \delta) \cdot(d \delta / d x)]=R^{1+3 \alpha_{2 T}+\alpha_{1 T}} \\
\times\left\{\Psi_{2, i}-\left(\xi_{2, i} \Psi_{2, i}^{\prime} / 3\right) \cdot[1-(4 x / \delta) \cdot(d \delta / d x)]\right\} \\
R^{-2 \alpha_{1 T}} \Psi_{1, i}^{\prime}=R^{2 \alpha_{2 T}} \Psi_{2, i}^{\prime} \\
R^{-3 \alpha_{1 T}} \Psi_{1, i}^{\prime \prime}=R^{1+\alpha_{2 T}} \Psi_{2, i}^{\prime \prime}
\end{gathered}
$$

Приравнивая степени при $R$ в левых и правых частях (63)-(65), получим переопределенную систему трех уравнений относительно $\alpha_{1 T}$ и $\alpha_{2 T}$ :

$$
\left\{\begin{array}{l}
-\alpha_{1 T}=1+3 \alpha_{2 T}, \\
-\alpha_{1 T}=\alpha_{2 T} \\
-3 \alpha_{1 T}=1+\alpha_{2 T}
\end{array}\right.
$$

Для выбора двух уравнений из трех применим полученное выше условие (62). Этому условию удовлетворяет только решение 2-го и 3-го уравнений системы (66):

$$
\alpha_{1 T}=-1 / 2, \quad \alpha_{2 T}=1 / 2
$$

С учетом (60) и (61) получаем следующие значения $\alpha_{1 \Psi}$ и $\alpha_{2 \Psi}$ :

$$
\alpha_{1 \Psi}=1 / 2, \quad \alpha_{2 \Psi}=3 / 2,
$$

а выражения (55) для $\left(\xi_{1}, \xi_{2}, \Psi_{1}, \Psi_{2}\right)$ принимают вид

$$
\begin{array}{ll}
\eta_{1}=R^{-1 / 2} \xi_{1}, & \eta_{2}=R^{1 / 2} \xi_{2}, \\
f_{1}=R^{1 / 2} \Psi_{1}, & f_{2}=R^{3 / 2} \Psi_{2} .
\end{array}
$$

С учетом (69) и в пренебрежении малыми слагаемыми система уравнений (56)-(59) принимает вид

$$
\begin{gathered}
\Psi_{1}^{\prime \prime \prime}+\Psi_{1} \Psi_{1}^{\prime \prime}-(2 / 3) \cdot\left(\Psi_{1}^{\prime}\right)^{2}=0, \\
\Theta_{1}^{\prime \prime}+\operatorname{Pr}_{1} \Psi_{1} \Theta_{1}^{\prime}=0, \\
\Psi_{2}^{\prime \prime \prime}+1=0, \\
\Theta_{2}^{\prime \prime}=0 .
\end{gathered}
$$

Связь $\eta_{1, i}$ и $\eta_{2, i}(43)$ преобразуется к виду

$$
\xi_{1, i}=\sqrt{v_{2} / v_{1}} \cdot R \xi_{2, i}=\left(\mu_{2} / \mu_{1}\right) \cdot \xi_{2, i} .
$$

При этом из (43) и (69) следует выражение для толщины паровой пленки $\delta(x)$ :

$$
\xi_{1, i} \approx 0
$$

При этом из (43) и (74) следует выражение для толщины паровой пленки $\delta(x)$ :

$$
\delta=R^{1 / 2} \Lambda^{3 / 4} x^{1 / 4} \xi_{2, i} .
$$

Граничные условия:

1) поверхность нагрева $\left(\xi_{2}=0\right)$ :

$$
\Psi_{2, w}=0, \quad \Psi_{2, w}^{\prime}=0, \quad \Theta_{2, w}=1
$$

2) межфазная граница $\left(\xi_{1}=\xi_{2} \approx 0\right.$ и $\left.\xi_{2}=\xi_{2, i}\right)$ :

условия равенства касательных к границе раздела фаз составляющих скоростей (64) и напряжений (65) примут простой вид соответственно:

$$
\Psi_{1, i}^{\prime}=\Psi_{2, i}^{\prime} ; \quad \Psi_{1, i}^{\prime \prime}=\Psi_{2, i}^{\prime \prime}
$$

Условие равенства массовых потоков (63) с учетом (75) примет вид

$$
\begin{aligned}
& \Psi_{1, i}-\left(\xi_{1, i} \Psi_{1, i}^{\prime} / 3\right) \cdot[1-(4 x / \delta) \cdot(d \delta / d x)]=R^{2} \\
& \times\left\{\Psi_{2, i}-\left(\xi_{2, i} \Psi_{2, i}^{\prime} / 3\right) \cdot[1-(4 x / \delta) \cdot(d \delta / d x)]\right\} \\
& \Rightarrow \Psi_{1, i} \approx 0 .
\end{aligned}
$$

Тепловое граничное условие (50) с учетом (76) принимает вид

$$
\begin{aligned}
& \left(K_{1} / \operatorname{Pr}_{1}\right) \cdot \Theta_{1, i}^{\prime}-\left(K_{2} / \operatorname{Pr}_{2}\right) \cdot \Theta_{2, i}^{\prime}+R^{1 / 2} B \chi^{1 / 4} \\
& =R^{2} \cdot\left[\Psi_{2, i}+(4 / 3) \cdot \chi \Psi_{2, i}^{\prime} \cdot\left(d \xi_{2, i} / d \chi\right)\right]
\end{aligned}
$$


где введена безразмерная координата $\chi=x / L$.

Температурные граничные условия (53) остаются без изменений.

3) объем жидкости $\left(\xi_{1} \rightarrow \infty\right)$ :

$$
\Psi_{1, \infty}^{\prime}=0, \quad \Theta_{1, \infty}=0 .
$$

Полученная система уравнений и граничных условий - общая как для случая малых недогревов (жидкости, близкой к насыщению), так и для сильно недогретых жидкостей. И хотя данная система по-прежнему не автомодельна относительно координаты $x$, она вполне поддается аналитическому решению.

Решение уравнения (72), удовлетворяющее условиям (77), имеет вид

$$
\Psi_{2}=-\xi_{2}^{3} / 6+A \xi_{2}^{2}, \quad \Psi_{2}^{\prime}=-\xi_{2}^{2} / 2+2 A \xi_{2},
$$

где $A$ не зависит от $\xi_{2}$, но может зависеть от других параметров задачи.

Решение уравнения (73), удовлетворяющее условиям (53) и (77), имеет вид

$$
\Theta_{2}=1-\xi_{2} / \xi_{2, i}, \quad \Theta_{2}^{\prime}=-1 / \xi_{2, i} .
$$

Перед получением решения уравнения движения жидкости (70) несколько переопределим граничное условие на межфазной границе, положив, что имеет место равенство

$$
\Psi_{1, i}^{\prime}=C^{2},
$$

где константа $C$ в (84) будет определена ниже из условий сопряжения решения для жидкости с решением для парового слоя.

Приближенным решением уравнения (70) с граничным условием (84) является

$$
\Psi_{1}=C \cdot\left[1-\exp \left(-C \xi_{1}\right)\right] .
$$

Выражение (85) дает отклонение от численного решения уравнения (70), не превышающее по абсолютной величине $4 \%$ во всем диапазоне изменения $\xi_{1}$.

Уравнение (71) с учетом (85) и граничных условий (53), (81) имеет решение

$$
\begin{aligned}
\Theta_{1}= & 1-\int_{0}^{\gamma} \exp \left[-P r_{1} \gamma^{\prime}-P r_{1} \exp \left(-\gamma^{\prime}\right)+P r_{1}\right] d \gamma^{\prime} \\
& / \int_{0}^{\infty} \exp \left[-P r_{1} \gamma-P r_{1} \exp (-\gamma)+P r_{1}\right] d \gamma,
\end{aligned}
$$

где введены обозначения $\gamma=C \xi_{1}, \gamma^{\prime}=C \xi_{1}^{\prime}$.

Простые вычисления показывают, что стоящий в знаменателе (86) интеграл

$$
I\left(P r_{1}\right)=\int_{0}^{\infty} \exp \left[-P r_{1} \gamma-P r_{1} \exp (-\gamma)+P r_{1}\right] d \gamma
$$

при $P r_{1} \ll 1$ равен $I\left(P r_{1}\right) \approx 1 / P r_{1}$, а при $\operatorname{Pr}_{1} \gg 1-$ $I\left(P r_{1}\right) \approx \sqrt{\pi /\left(2 P r_{1}\right)}$. Для всего диапазона чисел $P r_{1}$ в качестве выражения для этого интеграла можно использовать следующую интерполяцию:

$$
I\left(P r_{1}\right) \approx \sqrt{1 / \operatorname{Pr}_{1}^{2}+\pi /\left(2 P r_{1}\right)} .
$$

Численным интегрированием (87) установлено, что интерполяция (88) хорошо описывает предельные случаи $\operatorname{Pr}_{1} \ll 1$ и $\operatorname{Pr}_{1} \gg 1$, но несколько занижает значение $I\left(P r_{1}\right)$ при промежуточных значениях аргумента $\left(0.1<P r_{1}<10\right)$, максимальное занижение не превышает $8 \%$.

Из (86) для производной $\Theta_{1, i}^{\prime}$ имеем

$$
\Theta_{1, i}^{\prime}=-C / I\left(\operatorname{Pr}_{1}\right) \text {. }
$$

Из граничных условий (78) с учетом (82) и (85) получим

$$
\left\{\begin{array}{l}
C^{2}=-\xi_{2, i}^{2} / 2+2 A \xi_{2, i}, \\
C^{3}=\xi_{2, i}-2 A .
\end{array}\right.
$$

Из определения $\xi_{2}$ (2-я формула (69)) и малости $R$ следует, что $\xi_{2, i}$ велико. Используя это, можно получить приближенное решение системы (90)

$$
A=\xi_{2, i} / 4+1 /\left(4 \xi_{2, i}\right)^{2 / 3} ; \quad C=\left(\xi_{2, i} / 2\right)^{1 / 3}-1\left(3 \xi_{2, i}\right),
$$

где 2-е слагаемые в правых частях выражений - малые добавки к 1-м слагаемым. Из (91) видим, что как $A$, так и $C$ зависят от $\xi_{2, i}$.

Подставляя полученные значения констант $A$ и $C$ в (86) и (89), получим выражения для $\Psi_{2, i}, \Psi_{2, i}^{\prime}$ и $\Theta_{1, i}^{\prime}$ :

$$
\begin{gathered}
\Psi_{2, i}=\xi_{2, i}^{3} / 12+\left(\xi_{2, i} / 2\right)^{4 / 3} \approx \xi_{2, i}^{3} / 12, \\
\Psi_{2, i}^{\prime}=-\xi_{2, i}^{2} / 2+\left(\xi_{2, i}^{2} / 4+1 /\left(4 \xi_{2, i}\right)^{2 / 3}\right) \cdot 2 \xi_{2, i} \\
=\left(\xi_{2, i} / 2\right)^{1 / 3} \\
\Theta_{1, i}^{\prime}=-\left(\left(\xi_{2, i} / 2\right)^{1 / 3}-1 /\left(3 \xi_{2, i}\right)\right) / I\left(\operatorname{Pr}_{1}\right) \\
\approx-\left(\xi_{2, i} / 2\right)^{1 / 3} / I\left(\operatorname{Pr}_{1}\right) .
\end{gathered}
$$

В итоговых выражениях для $\Psi_{2, i}$ и $\Theta_{1, i}^{\prime}$ пренебрегли малыми добавками, обусловленными большой величиной $\xi_{2, i}$, а в выражении для производной $\Psi_{2, i}^{\prime}$ осталось лишь слагаемое, соответствующее именно малой добавке к $A$.

Подставим выражения (92)-(94) для $\Psi_{2, i}, \Psi_{2, i}^{\prime}, \Theta_{1, i}^{\prime}$ и (83) для $\Theta_{2, i}^{\prime}$ в условие $(80)$ :

$$
\begin{aligned}
& -K_{1} \xi_{2, i}^{1 / 3} /\left(2^{1 / 3} \operatorname{Pr}_{1} I\left(\operatorname{Pr}_{1}\right)\right)+K_{2} /\left(\operatorname{Pr}_{2} \xi_{2, i}\right)+R^{1 / 2} B \chi^{1 / 4} \\
& =R^{2} \cdot\left[\xi_{2, i}^{2} / 12+\left(2^{5 / 3} / 3\right) \cdot \xi_{2, i}^{1 / 3} \chi \cdot\left(d \xi_{2, i} / d \chi\right)\right] .
\end{aligned}
$$

В результате получено обыкновенное дифференциальное уравнение 1-го порядка относительно $\xi_{2, i}(\chi)$. 
Предположим, что при $\chi \ll 1$ имеет место степенная зависимость $\xi_{2, i}=\xi_{P} \chi^{P}$. Достаточно легко понять, что при положительных $P$ самым большим слагаемым по порядку величины является 2-е слагаемое в левой части (95), т.е. слагаемое, ответственное за теплопроводность в паровой пленке. При этом (95) вырождается в бессодержательное выражение, так как в нем остается только это слагаемое. При отрицательном $P$ самым большим слагаемым станет 1-е слагаемое в правой части (95), и это также превратит данное уравнение в бессодержательное, поскольку и в этом случае в нем останется только одно слагаемое. Единственный вариант, при котором (95) сохранит физический смысл равенство степени $P$ нулю, т.е. $\xi_{2, i}=$ const. Но при этом имеет место $\left(d \xi_{2, i} / d \chi\right)=0$, а само уравнение (95), упростившись, превратится из дифференциального в алгебраическое:

$$
\begin{aligned}
& R^{-2} \cdot\left[K_{2} /\left(\operatorname{Pr}_{2} \xi_{2, i}\right)-K_{1} \xi_{2, i}^{1 / 3} /\left(2^{1 / 3} \operatorname{Pr}_{1} I\left(\operatorname{Pr}_{1}\right)\right)\right] \\
& +R^{-3 / 2} B \chi^{1 / 4}=\xi_{2, i}^{3} / 12 .
\end{aligned}
$$

Предполагая далее, что и во всей области определения переменной $\chi$, т.е. от 0 до 1,2 -е слагаемое в правой части (95) если не равно нулю, то много меньше первого слагаемого, можно считать, что при $0 \leq \chi \leq 1$ данное граничное условие выполняется именно в форме (96), т. е. является алгебраическим уравнением.

Введем обозначения

$$
\begin{gathered}
z=\left(R^{2} \operatorname{Pr}_{2} /\left(12 K_{2}\right)\right)^{1 / 3} \cdot \xi_{2, i}^{4 / 3}, \\
S=6^{1 / 3}\left(K_{1} / \operatorname{Pr}_{1}\right) /\left[R^{2 / 3} I\left(\operatorname{Pr}_{1}\right) \cdot\left(K_{2} / \operatorname{Pr}_{2}\right)^{2 / 3}\right] .
\end{gathered}
$$

Отметим, что комплекс $S$ можно назвать критерием, параметром „недогрева“, так как он пропорционален недогреву жидкости $K_{1}$.

Тогда, упорядочивая слагаемые (98) по убыванию степеней при $z$, получим

$$
z^{3}+S z-12^{1 / 4} B \cdot\left(P r_{2} / K_{2}\right)^{3 / 4} \cdot \chi^{1 / 4} z^{3 / 4}-1=0 .
$$

\section{4. Условие автомодельности задачи по физической координате $x$}

В выражении (99) от безразмерной координаты $\chi$ (и от физической координаты $x$ ) зависит только одно 3 -е слагаемое, „радиационное“. Таким образом, если при решении задачи этим слагаемым по какой-либо причине пренебрегается (например, при относительно небольших перегревах поверхности нагрева), то задача в целом становится автомодельной по физической координате $x$.

Отметим также, что при этом введенная в (97) переменная $z$, а значит, и $\xi_{2, i}$, не зависят от $\chi$. То есть в (95) всегда имеет место $\left(d \xi_{2, i} / d \chi\right)=0$, и это уравнение всегда имеет вид (96) во всем диапазон изменения координаты $\chi$.
Без радиационного слагаемого уравнение (99) представляет собой кубическое уравнение относительно $z=z_{0}$ :

$$
z_{0}^{3}+S z_{0}-1=0
$$

Уравнение (100) имеет единственный вещественный корень:

$$
\begin{aligned}
z_{0}= & z_{0}(S)=\sqrt[3]{\sqrt{(S / 3)^{3}+1 / 4}+1 / 2} \\
& -\sqrt[3]{\sqrt{(S / 3)^{3}+1 / 4}-1 / 2}
\end{aligned}
$$

Для $z_{0}(S)$ имеет место: 1$)$ при $S \ll 1 z_{0} \approx 1$ и 2) при $S \gg 1 z_{0} \approx 1 / S$.

Далее, используя связь (97) и выражения (83), (94), возможно получить выражения для критериев Нуссельта для теплообмена в паровом слое и в жидкости. Но проделаем это ниже, сразу с учетом теплообмена излучением.

\section{5. Учет теплообмена излучением}

Естественно, что при этом задача теряет автомодельность по физической координате $x$.

Влияние радиационного теплового потока можно учесть, решая уравнение (99) с помощью методов теории возмущения, в предположении, что радиационное слагаемое является малой добавкой к решению без учета теплового излучения $\left(z_{0}(S)\right)$ :

$$
z(S) \approx z_{0}(S)+\delta z\left(S, B \cdot\left(\operatorname{Pr}_{2} / K_{2}\right)^{3 / 4} \chi^{1 / 4}\right), \quad|\delta z| \ll z_{0} .
$$

Тогда решение уравнения (99) имеет вид

$$
\begin{aligned}
& z \approx z_{0}(S)+12^{1 / 4} B \cdot\left(P r_{2} / K_{2}\right)^{3 / 4} \chi^{1 / 4} \\
& \times\left[z_{0}^{3 / 4}(S) /\left(3 z_{0}^{2}(S)+S\right)\right],
\end{aligned}
$$

а выражение для $\xi_{2, i}$ с учетом (97) будет иметь вид

$$
\begin{aligned}
\xi_{2, i}= & {\left[12 K_{2} /\left(\operatorname{Pr}_{2} R^{2}\right)\right]^{1 / 4} z_{1}(S) } \\
& \times\left[1+2^{1 / 2} B z_{2}(S) \chi^{1 / 4} /\left(3 K_{2} / P r_{2}\right)^{3 / 4}\right]^{3 / 4} .
\end{aligned}
$$

В (104) введены две функции от $S$ :

$$
z_{1}(S)=z_{0}^{3 / 4}(S), \quad z_{2}(S)=3 /\left[z_{0}^{1 / 4}(S) \cdot\left(3 z_{0}^{2}(S)+S\right)\right] .
$$

Вид функций $z_{1}(S)$ и $z_{2}(S)$ выбран так, чтобы при $S \rightarrow 0$ (т.е. в насыщенной жидкости) имело место $z_{1} \rightarrow 1$ и $z_{2} \rightarrow 1$.

Критерий Нуссельта для теплообмена в жидкости и в паровом слое, как функцию от физической координаты $x$, можно определить следующим образом:

$$
\begin{aligned}
& N u_{1}(x)=\alpha_{1}(x) L / \lambda_{1}=q_{1, i}(x) L /\left[\lambda_{1} \cdot\left(T_{s}-T_{\infty}\right)\right] \\
& =-L /\left(T_{s}-T_{\infty}\right) \cdot\left(\partial T_{1} / \partial y\right)_{i},
\end{aligned}
$$




$$
\begin{aligned}
& N u_{2}(x)=\alpha_{2}(x) L / \lambda_{2}=q_{2, i}(x) L /\left[\lambda_{2} \cdot\left(T_{w}-T_{s}\right)\right] \\
& =-L /\left(T_{w}-T_{s}\right) \cdot\left(\partial T_{2} / \partial y\right)_{i}
\end{aligned}
$$

После подстановки обозначений, полученных связей $(33),(36),(69),(83),(94)$ и решения (104) в (106) и (107), выражения для критерия Нуссельта приобретают вид

$$
\begin{aligned}
& N u_{1}(\chi)=\frac{3^{1 / 3}}{2^{2 / 3} I\left(P r_{1}\right)} \cdot\left(\frac{\rho_{1}}{\rho_{2}}\right)^{1 / 4} \cdot R^{1 / 3} A r_{1}^{1 / 4}\left(\frac{K_{2}}{P r_{2}}\right)^{1 / 12} \\
& \times z_{0}^{1 / 4}(S) \chi^{-1 / 4} \cdot\left[1+\frac{2^{1 / 2} B z_{2}(S) \chi^{1 / 4}}{3^{3 / 4}\left(K_{2} / P r_{2}\right)^{3 / 4}}\right]^{1 / 4}, \\
& N u_{2}(\chi)=\left(\frac{P r_{2}}{K_{2}} A r_{2}\right)^{1 / 4} \cdot\left(2 \chi^{1 / 4} z_{1}(S)\right)^{-1} \\
& \times\left[1+\frac{2^{1 / 2} B z_{2}(S) \chi^{1 / 4}}{3^{3 / 4}\left(K_{2} / P r_{2}\right)^{3 / 4}}\right]^{1 / 4}
\end{aligned}
$$

где введены обозначения для чисел Архимеда жидкости и пара

$$
A r_{j}=\Delta \rho g L^{3} /\left(\rho_{j} v_{j}^{2}\right), \quad j=1,2 .
$$

Найдем средний по межфазной поверхности критерий Нуссельта для жидкости и средний по поверхности нагрева - для пара. Осреднение сводится к интегрированию по безразмерной переменной $\chi$ от 0 до 1 . Выражения (108) и (109) поддаются непосредственному интегрированию, но вид полученных решений достаточно сложен. Поэтому для упрощения интегрирования и его результата учтем малость вторых слагаемых в прямых скобках выражений (108) и (109) - линеаризуем (108) и (109) относительно этих слагаемых. После чего результат осреднения/интегрирования будет иметь достаточно простой вид:

$$
\begin{aligned}
N u_{1} \approx & \frac{2^{4 / 3}}{3^{2 / 3} I\left(P r_{1}\right)} \cdot\left(\frac{\rho_{1}}{\rho_{2}}\right)^{1 / 4} \cdot R^{1 / 3} A r_{1}^{1 / 4}\left(\frac{K_{2}}{P r_{2}}\right)^{1 / 12} \\
& \times z_{0}^{1 / 4}(S) \cdot\left[1+\frac{3^{1 / 4} B z_{2}(S)}{2^{7 / 2}\left(K_{2} / P r_{2}\right)^{3 / 4}}\right], \\
N u_{2} \approx & \frac{2}{3 z_{1}(S)} \cdot\left(\frac{P r_{2}}{K_{2}} A r_{2}\right)^{1 / 4} \cdot\left[1-\frac{3^{5 / 4} B z_{2}(S)}{2^{7 / 2}\left(K_{2} / P r_{2}\right)^{3 / 4}}\right]
\end{aligned}
$$

$$
\text { где } \quad N u_{j}=\left\langle\alpha_{j}\right\rangle \cdot L / \lambda_{i}, \quad j=1,2
$$

- средние по поверхности критерии Нуссельта.

Из (111) и (112) видно, что тепловое излучение интенсифицирует конвективный теплообмен между межфазной поверхностью и объемом жидкости, но снижает критерий Нуссельта для теплообмена теплопроводностью в паровом слое.

Найдем предельные при малых (равных нулю) и больших значения параметра недогрева $S$ выражения для критерия Нуссельта:
— теплообмен от межфазной поверхности в жидкость:

1) близкая к насыщению жидкость $(S \ll 1)$ :

$$
\begin{aligned}
N u_{1}= & \frac{2^{4 / 3}}{3^{2 / 3} I\left(\operatorname{Pr}_{1}\right)} \cdot\left(\rho_{1} / \rho_{2}\right)^{1 / 4} R^{1 / 3} A r_{1}^{1 / 4}\left(\frac{K_{2}}{\operatorname{Pr}_{2}}\right)^{1 / 12} \\
& \times\left[1+\frac{3^{1 / 4} B}{2^{7 / 2}\left(K_{2} / P r_{2}\right)^{3 / 4}}\right]
\end{aligned}
$$

2) сильно недогретая жидкость $(S \gg 1)$ :

$$
\begin{aligned}
N u_{1} & =\frac{2^{5 / 4}}{3^{3 / 4}} \cdot\left[A r_{1} \cdot\left(\mu_{2} / \mu_{1}\right) \cdot \frac{\left(K_{2} / P r_{2}\right)}{\left(K_{1} / \operatorname{Pr}_{1}\right)} \cdot \frac{1}{I^{3}\left(\operatorname{Pr}_{1}\right)}\right]^{1 / 4} \\
& \times\left[1+\frac{3 R^{1 / 2} I^{3 / 4}\left(\operatorname{Pr}_{1}\right) B}{2^{15 / 4}\left(K_{2} / P r_{2}\right)^{1 / 4}\left(K_{1} / P r_{1}\right)^{3 / 4}}\right]
\end{aligned}
$$

- теплообмен между поверхностью нагрева и межфазной поверхностью:

1) близкая к насыщению $(S \ll 1)$ или просто насыщенная жидкость $(S=0)$ :

$$
N u_{2}=\frac{2}{3} \cdot\left(\frac{P r_{2}}{K_{2}} A r_{2}\right)^{1 / 4} /\left[1+\frac{3^{5 / 4} B}{2^{7 / 2}\left(K_{2} / P r_{2}\right)^{3 / 4}}\right]
$$

2) сильно недогретая жидкость $(S \gg 1)$ :

$$
\begin{gathered}
N u_{2}=\frac{2^{5 / 4}}{3^{3 / 4} R^{1 / 2} I^{3 / 4}\left(P r_{1}\right)} \cdot\left[\frac{\left(K_{1} / P r_{1}\right)}{\left(K_{2} / P r_{2}\right.}\right]^{3 / 4} \cdot A r_{2}^{1 / 4} \\
/\left[1+\frac{3^{2} R^{1 / 2} I^{3 / 4}\left(P r_{1}\right) B}{2^{15 / 4}\left(K_{2} / P r_{2}\right)^{1 / 4}\left(K_{1} / P r_{1}\right)^{3 / 4}}\right] .
\end{gathered}
$$

Отметим, что выражение для критерия Нуссельта по теплообмену между поверхностью нагрева и межфазной поверхностью в случае насыщенной жидкости (116) и в пренебрежении радиационным теплообменом фактически совпадает с формулой Бромли для пленочного кипения насыщенной жидкости на горизонтальном цилиндре [14]:

$$
N u_{2}=C_{B r} \cdot\left(P r_{2} A r_{2} / K_{2}\right)^{1 / 4}
$$

где безразмерный коэффициент лежит в пределах от 0.51 до 0.73 при среднем значении, равном 0.62 .

\section{Заключение}

Для задачи о пленочном кипении на вертикальной поверхности жидкости произвольного недогрева показана эффективность методов автомодельности. При этом получен ряд новых результатов:

1 Показано наличие полной автомодельности (автомодельности 1-го рода) по параметру соотношения подъемных сил в жидкости и в паре и, как следствие, отсутствие влияния на решение сил плавучести в жидкости.

2. Показано наличие неполной автомодельности (автомодельности 2-го рода) по параметру $R=\left(\rho_{2} / \rho_{1}\right) \cdot \sqrt{v_{2} / v_{1}}$. 
3. Показано наличие автомодельности по физической координате вдоль поверхности нагрева (по координате $x$ ) в случае пренебрежения тепловым потоком излучения.

4. С использованием свойств указанных выше автомодельностей получено приближенное аналитическое решение задачи для произвольного недогрева теплоносителя с учетом теплового излучения с поверхности нагрева.

5. Получены выражения для критерия Нуссельта для теплообмена в паровом слое, между поверхностью нагрева и межфазной поверхностью, и от межфазной поверхности в объем жидкости в условиях произвольного недогрева жидкости с учетом теплового излучения с поверхности нагрева.

6. Получены предельные выражения для указанных в предыдущем пункте Заключения критериев Нуссельта для условий насыщения и для сильно недогретой жидкости.

В заключении необходимо добавить, что сопоставление с экспериментальными данными и рекомендации по использованию полученных соотношений авторы планируют изложить в последующих публикациях.

\section{Финансирование работы}

Работа выполнена за счет собственных средств ФГУП „Научно-исследовательский технологический институт им. А.П. Александрова“.

\section{Конфликт интересов}

Авторы заявляют, что у них нет конфликта интересов.

\section{Список литературы}

[1] В.В. Ягов. Теплоэнергетика, 11, 16 (2019).

[2] В.В. Ягов, А.Р. Забиров, П.К. Канин, М.А. Денисов. ИФЖ, 90 (2), 287 (2017).

[3] В.В. Ягов. Вестник МЭИ, 6, 86 (2017).

[4] N.I. Kolev. Multiphase Flow Dynamics 3. (Springer, Heidelberg, Berlin, 2011), p. 245.

[5] С.Л. Соловьев. Тепловые процессы в технике. Изд. МАИ (НИУ), 2 (5), 197 (2010).

[6] А.А. Гухман. Физические основы теплопередачи. T.I. Теория подобия и ее приложения (Госэнергоиздат, Л., M., 1934)

[7] А.А. Гухман. Введение в теорию подобия (Высшая школа, М., 1973).

[8] Я.Б. Зельдович, Ю.П. Райзер. Физика ударных волн и высокотемпературных гидродинамических явлений (Физматгиз, М., 1963)

[9] К.В. Брушлинский, Я.М. Каждан. УМН, 18 (2 (110)), 3 (1963).

[10] Г.И. Баренблатт, Я.Б. Зельдович. УМН, 26 (2 (158)), 115 (1971).

[11] Г.И. Баренблатт. Подобие, автомодельность, промежуточная асимптотика (Гидрометеоиздат, Л., 1978).

[12] K.Nishikawa, T. Ito. Int. J. Heat Mass Transfer., 9, 103 (1966).
[13] J.C.Y. Koh, E.M. Sparrow, J.P. Hartnett. Int. J. Heat Mass Transfer., 2, 69 (1961).

[14] L.A. Bromley. Chem. Engineer. Progress, 46, 221 (1950). 